\title{
New synthetic 3D culture systems to unlock the future of organoids in research and therapy
}

\author{
"Today, we are at the dawn of the era of organoids..."
}

First draft submitted: 26 June 2017; Accepted for publication: 12 July 2017; Published online: 15 September 2017

Keywords: 3D synthetic hydrogels $\bullet$ drug development $\bullet$ extracellular matrix $\bullet$ organoids - personalized medicine

Ever since the times of Aristotle and Galen, human beings have been fascinated by the intricate mechanisms that govern the biology of the human body. The vast complexity of nature, however, has made it challenging to unravel the minute mysteries that govern how organisms develop and function. Today, we are at the dawn of the era of organoids, which potentially hold the ability to revolutionize our understanding of tissue and disease development as well as to open the door to personalized medical treatments.

Organoids are miniature organ-like structures that are grown in vitro in $3 \mathrm{D}$ environments. They are derived from one or few cells extracted from a tissue, and when cultured ex vivo in appropriate conditions, they self-organize and grow to adopt key structural and functional properties of the target organ. Since they can be generated from patient biopsies, organoids capture many of the specific disease features of the patient - something that traditional cell culture techniques often struggle to accomplish. That is why $3 \mathrm{D}$ organoid culture is considered one of the most exciting advances in the life sciences.

Although the technology for growing organoids has rapidly improved in recent years, today's methods find their origin in 1906 when Ross Harrison pioneered experiments on the origin of nerve fibers [1]. In his research, Harrison isolated a fragment of embryo nerve cord and placed it on a drop of lymph, thus providing an adequate environment that allowed the tissue to grow. Since then, other researchers have adapted Harrison's system to culture diverse types of cells in vitro. However, it was not until 1956 that Robert Ehrmann and George Gey published the first method to prepare collagen gels for culturing cell lines on 2D Petri dishes [2]. Later in 1977, Richard Swarm and his group discovered and defined the laminin-rich gel that is still used today as common laboratory procedure [3].

As model systems improved, it became more and more evident that the extracellular matrix (ECM) has the ability to stimulate complex cell behaviors and influence gene expression. In 1975, Michalopoulos and Pitot observed that it was possible to induce differentiation of some specific cells just by modifying the characteristics of the substratum to which they were attached [4], and in 1982 the article titled 'How does ECM regulate gene expression?' proposed that the ECM could alter gene expression by exerting physical and chemical influences on the biochemistry of the cell [5]. Today, research is addressing how stem cell identity is defined by the delicate balance in the

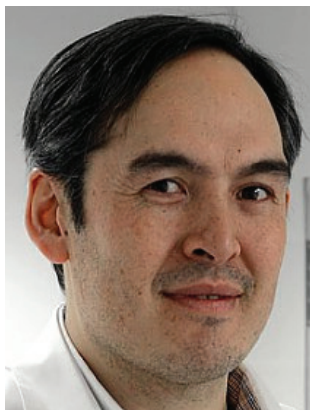

Colin Sanctuary

QGel SA, Innovation Park, EPFL Building G, 1015 Lausanne, Switzerland Tel.: +41216940707 Fax: +41215604957 info@qgelbio.com 
niche that surrounds it, integrating cell-intrinsic and cell-extrinsic factors for proper tissue homeostasis [6].

As it became accepted that functional differentiation is at least partially dependent on 3D architecture, a growing body of evidence has shown that cells cultured in $2 \mathrm{D}$ are not always representative of the in vivo situation. Traditional $2 \mathrm{D}$ cultures often fail to reproduce the complex spatial morphology observed in vivo as they bear little physical, molecular or physiological similarity to the tissue of origin [7].

Biologists and bioengineers have started investigating 3D hydrogel matrices that would recapitulate aspects of the native microenvironment for in vitro cell culture. This has led to a shift from 2D cultures to complex extracellular 3D animal-derived matrices, which have become the 3D culture matrix of choice for organoids. Unfortunately, natural animal-derived ECMs have undefined compositions, making it difficult to pinpoint which specific factors present in the microenvironment are having what effect on the cells. Now, a new generation of synthetic 3D matrices based on modular hydrogel cross-linked networks is able to tune key parameters of the ECM (such as stiffness properties, ECM degradability and the ability to tether specific cell adhesion sites to the matrix) enabling researchers to culture any cell type, including organoids [8,9].

These specialized synthetic and tunable ECMs

\section{"Organoids are miniature organ-like structures that are grown in vitro in 3D environments.}

bring a novel method to create unique structural, biological and biochemical environments to support the physiologically relevant culture of any cell type. In vivo, ECM compositions differ vastly depending on the cell types they surround. For example, the ECM of cartilage is dramatically different than the ECM of brain tissue. The presence of specific growth factors and cytokines as well as mechanical forces existing in the ECM can act as modulators of cell proliferation and differentiation - ultimately determining the healthy or diseased state of a tissue. Synthetic hydrogels allow the precise control of key parameters of the ECM, and are thus capable of providing biologically accurate model systems suitable for many cell-based applications. Scientists can now purchase a variety of validated synthetic ECMs for many cell types including: brain, breast, colon, kidney, lung, ovary, pancreas, placenta, prostate and skin. If no ECM has been validated yet for a specific application, libraries of existing ECMs of defined composition can be screened to find the appropriate ECM and culture condition combinations to support the culture of any cell type, including organoids.

We have just started unlocking the full potential of organoid technology and the application of 3D synthetic ECMs is already yielding results to advance fundamental research as well as for clinical research. Recently, the use of synthetic hydrogel networks has allowed scientists to define the key ECM parameters that govern organoid formation, with separate stages of the process requiring changing mechanical properties of the hydrogel [10]. Moving further, biologists will be empowered to explore the processes of organ development and differentiation more deeply on a molecular level. For the pharmaceutical industry, drug developers will be able to exploit the use of synthetic hydrogels to establish organoid cultures as a reliable and scalable tool to test a wide variety of drugs and compounds as well as design drug regimens in conjunction with other diagnostic and prognostic factors. For clinicians, the organoid technology has the potential to introduce a novel practice based on functional and patient-specific tests to select personalized treatments and dosages for a particular disease.

Looking ahead, organoids may well bridge the gap between the preclinic and the clinic and finally open the gateway to harnessing the full potential of personalized medicine. That is why today's efforts are focused on developing easy-to-manage and ready-touse hydrogel models to grow organoids from every patient-derived biopsy. Making organoids available for routine use in hospitals and clinics will pave the way to improved diagnostics and personalized cancer treatments, as patients gain access to therapies tailored for their specific disease.

And with revolutionary genome-editing technologies, such as CRISPR/Cas9 making leaps from laboratory to industry, 3D organoid models will soon meet genome engineering and open to a whole new range of applications. With 3D synthetic ECMs, the field of organoids is now starting to blossom.

\section{Financial \& competing interests disclosure}

C Sanctuary is Chief Executive Officer, Board Member \& Founder of QGel. The author has no other relevant affiliations or financial involvement with any organization or entity with a financial interest in or financial conflict with the subject matter or materials discussed in the manuscript apart from those disclosed.

No writing assistance was utilized in the production of this manuscript.

\section{Open access}

This work is licensed under the Attribution-NonCommercialNoDerivatives 4.0 Unported License. To view a copy of this license, visit http://creativecommons.org/licenses/by-nc-nd/4.0/ 


\section{References}

1 Harrison RG. Observations on the living developing nerve fiber. Exp. Biol. Med. 4, 140-143 (1906).

2 Ehrmann RL, Gey GO. The growth of cells on a transparent gel of reconstituted rat-tail collagen. J. Natl. Cancer Inst. 16, 1375-1403 (1956).

3 Orkin RW, Gehron P, McGoodwin EB, Martin GR, Valentine T, Swarm R. A murine tumor producing a matrix of basement membrane. J. Exp. Med. 145, 204-220 (1977).

4 Michalopoulos G, Pitot HC. Primary culture of parenchymal liver cells on collagen membranes. Morphological and biochemical observations. Exp. Cell Res. 94, 70-78 (1975).

5 Bissell MJ, Hall HG, Parry G. How does the extracellular matrix direct gene expression? J. Theor. Biol. 99, 31-68 (1982).
6 Kaplan RN, Psaila B, Lyden D. Niche-to-niche migration of bone-marrow-derived cells. Trends Mol. Med. 13, 72-81 (2007).

7 Wang F, Weaver VM, Petersen OW et al. Reciprocal interactions between $\beta 1$-integrin and epidermal growth factor receptor in three-dimensional basement membrane breast cultures: a different perspective in epithelial biology. Proc. Natl Acad. Sci. USA 95, 14821-14826 (1998).

8 Lutolf MP, Gilbert PM, Blau HM. Designing materials to direct stem-cell fate. Nature 462, 433-441 (2009).

9 Ranga A, Gobaa S, Okawa Y, Mosiewicz K, Negro A, Lutolf MP. 3D niche microarrays for systems-level analyses of cell fate. Nat. Commun. 5, 4324 (2014).

10 Gjorevski N, Sachs N, Manfrin A et al. Designer matrices for intestinal stem cell and organoid culture. Nature 539(7630), 560-564 (2016). 